\title{
Research on the Application of Dali Tie-dye Elements in the Design of Homestays
}

\author{
Xinyue Wang ${ }^{1, *}$ \\ ${ }^{1}$ Sichuan Fine Arts Institute, Chongqing, China \\ *Corresponding author. Email: 271755163@qq.com

\begin{abstract}
Dali is located in Yunnan Province in southwestern China. As a famous tourist city, there are a large number of homestays and various styles. There are many Bai tie-dye themed homestays in Dali, but the overall quality is not very high. This article mainly uses the literature research method and case analysis method to focus on the tie-dyeing skills of the Bai people in Dali, extracts its artistic elements, and analyzes the existing tie-dye themed homestays in Dali, in order to explore the design principles of ethnic cultural elements in the design of B\&B, to provide design strategies for related Dali tie-dye themed homestay design.
\end{abstract}

Keywords: Dali, Tie-dye, Homestay.

\section{INTRODUCTION: OVERVIEW OF TIE-DYE OF THE BAI NATIONALITY IN DALI}

Tie-dyeing is known as "wrapping" in ancient times. It is an ancient textile dyeing technique in China. Together with batik and hollow printing, it is known as the three ancient printing techniques in China. The tie-dyeing process of the Bai people in Dali is listed as the first batch of intangible cultural heritage in China because of its special skills, exquisite patterns and beautiful colors. At present, the areas that retain the tie-dyeing skills of the Bai people are located in western Yunnan Province, Weishan and Zhoucheng in Dali. Almost every local villager can use the traditional tie-dyeing skills, and Zhoucheng is also known as the "Tiedye Township". Dali tie-dyeing has very high aesthetic value and practical functions, but nowadays, there are fewer and fewer craftsmen who can do Dali tie-dyeing, and they are replaced by large-scale industrial production factories. The tie-dye products produced in batches in factories are generally shoddy. In order to improve efficiency and control the cost, modern tie-dye products are generally machine-printed and dyed with chemical dyes, but the effect is far less than that of craftsmen who use natural dyes. In addition, with the development of the Internet economy, the traditional tie-dyeing technique of the Bai people in
Dali will inevitably be impacted. The traditional tie-dyeing technique of Dali gradually fades out of people's sight, and its related exquisite crafts gradually lose their national charm.

\section{DALI TIE-DYE ELEMENT EXTRACTION}

\subsection{Color}

Most of the Dali tie-dye handicrafts are blue patterns on a white background or white patterns on a blue background. They are mainly composed of blue and white. In the Bai culture and customs, white represents white clouds and snowy mountains, and is a symbol of purity. Blue represents Erhai Lake. The blue sky and Cangshan mountains give people a natural and refreshing aesthetic feeling. The traditional tie-dye works of Dali are dyed with natural dyes. The dyes come from the blue indigo solution extracted from natural plants such as Radix Isatidis and Mugwort grown in Cangshan, Dali [1]. The dyed colors are unique and bright, and they will never fade. In addition to blue dyes, flowers and plants of various colors are also used as dyes in the tie-dyeing process, for example, using turmeric, safflower, and gardenia as dyes, the final tie-dye works of yellow, red, brown, purple, etc. can be obtained [2] ("Figure 1") In the process of dipdyeing, because the edges of the tied patterns are impregnated with dyes, there will be natural halos, 
so the tie-dyed patterns will produce a kind of nonreproducibility and uncertainty. The gradual
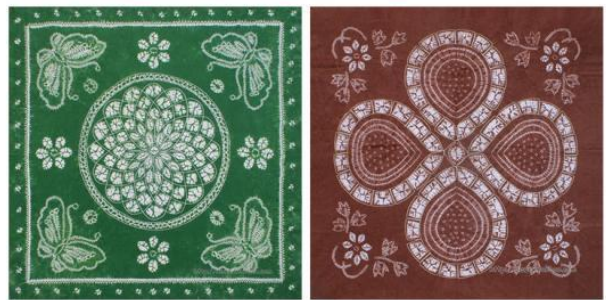

blending of colors gives people a feeling of misty clouds and mists.
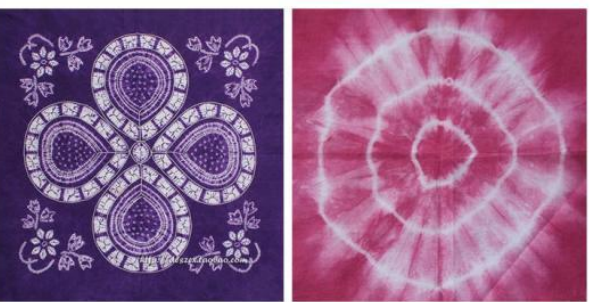

Figure 1 Tie-dye colors of Bai nationality in Dali (picture source: author's collation).

\subsection{Decorative Patterns}

The use of tie-dye patterns by the Bai people in Dali comes from their daily lives. They usually draw on the images of flowers, birds, trees, mountains, rivers, and animals in nature as decorative patterns. Among them, flower-shaped patterns appear most frequently in tie-dye handicrafts of the Bai nationality. The Bai people love plants very much, and potted plants can be seen everywhere in the courtyard of their homes. The most common plants of the Bai people in Dali are camellia, lantana, plum, orchid, etc. [3] They are good at discovering beautiful patterns in nature, subjectively transforming them, permuting and combining them to create symmetrical shapes. ("Figure 2") Besides, under the influence of religion and society, there are also figures and architectural symbols. In Tian Shun's "Research on the Architectural Image in the Tie-Dye Craft of the Bai Nationality", it is clearly pointed out that the Bai people draw on the architectural image of the traditional Bai nationality's dwellings in the process, of extracting the tie-dye pattern, such as the architectural image of the plum windows, mirror walls and other architectural components [4].
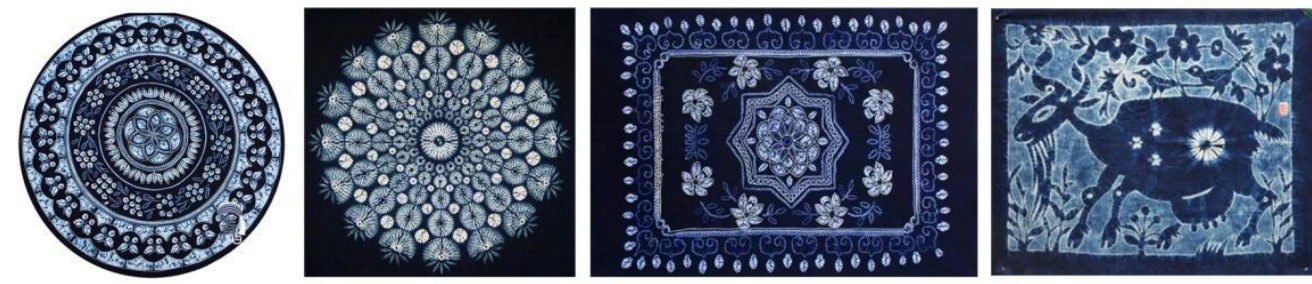

Figure 2 Tie-dye patterns of Bai nationality in Dali (picture source: finished by the author).

\subsection{Tie Flower and Dip Dyeing Process}

In addition to the visual charm presented by tiedye artworks, tie-dyeing techniques are also part of Dali tie-dyeing culture. The key part of the craftsmanship is the crystallization of national wisdom and local culture, and is an important element of Dali tie-dyeing. The process is mainly divided into the following steps: tying flowers, dyeing cloth, cooking, stitching, drying and ironing [5]. As the first and most important step of the whole process, tying flowers is also the most important step. Tie-up is to tie the white cloth with different stitches to form a knot, so that the tied part is not impregnated with dye. There are many types of tying techniques, and the final pattern will be different according to the different stitches. Traditional tying techniques include folding tying, splinting tying, free tying, flat needle tying, etc. [6].
Dyeing cloth is also one of the most important processes in Bai tie-dyeing. The whiteness of the cloth surface can be determined according to the degree of impregnation to create a gradual effect. At the same time, depending on the number of times of dyeing, the depth of the cloth color can be determined, and the method of dyeing directly determines the final effect of the work.

\section{CURRENT STATUS AND PROBLEMS OF DALI TIE-DYE THEME HOMESTAYS}

Dali Prefecture in Yunnan is rich in natural, historical, and cultural resources. Now it has developed into an important area for the development of tourism in our country, and the homestay industry has quickly become popular as a result. Although the homestay industry market in Dali is huge and diverse, due to factors such as 
insufficient supervision of homestay operators by relevant departments and lack of aesthetic standards for homestay design, the quality of local themed homestays is not uniform. Two tie-dye themed homestays of the Bai nationality in Dali, Yunnan are more typical examples ("Figure 3"). It can be seen from the picture that the indoor space lacks tie-dye cultural atmosphere, which is embodied in three aspects: the lack of tie-dye artistic atmosphere in the space environment; the single use of tie-dye elements; the lack of space for tie-dye cultural experience.

First of all, the current tie-dye themed homestays generally lack a sense of space and atmosphere, and the creation of the sense of atmosphere is mainly reflected in the selection of colors and materials. In the two selected homestays, the selection and collocation of furniture are not exquisite, there is no main color tone in the space, and the collocation of furniture materials makes the space look stiff. The matching of dark wooden furniture, wooden floors, white walls, mosaic tiles, and glass curtain walls is inconsistent. The interior furnishings have nothing to do with Dali's customs and tie-dyeing culture. The space style lacks uniformity and does not reflect the cultural atmosphere and decorative effects of tie-dyeing art.

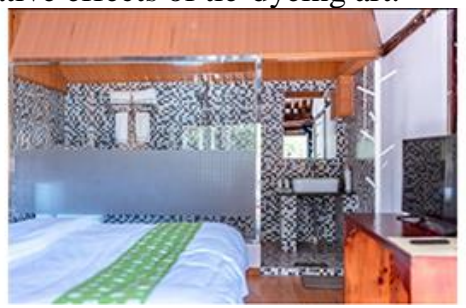

Secondly, in the indoor living space and public space of a homestay, the decorative patterns and expression methods of tie-dye elements are single, and usually only curtains, beddings, hanging paintings and other products with tie-dye patterns and colors are used for partial decoration and decoration. The technique is monotonous and flowing on the surface, making the space thin and not enough to have a vivid and three-dimensional visual effect.

Last but not least, the space function does not meet the needs of tourists to experience the local ethnic culture and customs. The guesthouse only provides tourists with housing, dining, and leisure services, and the space function is single. The public space lacks a visit and experience area for the Dali tie-dye production process, and lacks the sense of participation and interaction between tourists and the space, so that the emotional communication and exchange between the space and the tourists cannot be achieved. This type of homestay design is generally due to the designer's lack of investigation and research on local regional culture and folk customs, and no in-depth exploration of local cultural characteristics and customs, so it is not a real homestay design.

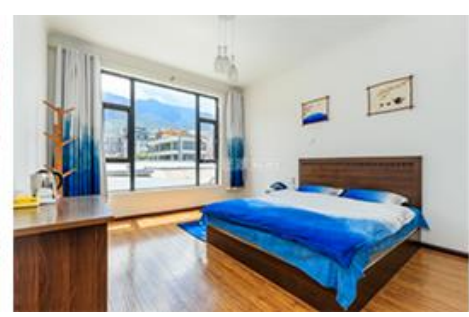

Figure 3 A tie-dye theme homestay with characteristics of Bai nationality in Dali (photo source: Internet).

\section{THE DESIGN PRINCIPLES OF TIE-DYE ELEMENTS IN THE DESIGN OF HOMESTAYS}

\subsection{Principles of Space Atmosphere Creation}

The design of homestays should highlight the local cultural value and highlight its cultural symbols. To create an environmental atmosphere in the space, it is necessary to rely on the traditional residential buildings of the Bai nationality, and with the unique nationality, life, and local unique temperament of the residential buildings, fully highlight the characteristics of the tie-dyeing skills of the Bai nationality in Dali, so that it is better to show and inherit Dali tie-dye in the design of homestays. At the same time, the unity and coordination of the overall style should be fully considered in terms of colors, materials, and indoor lighting. First of all, the color of the space is the key to integrating the style of the space theme. Dali tie-dye is mostly blue and white. The author uses blue and white to design the interior space, using cloth art with tie-dye elements, vases, teapots, etc. The lamps and hanging paintings echo the colors, and a small range of warm colors and indoor environment are added to render the tie-dye artistic atmosphere. ("Figure 4") The reasonable selection of materials can also play a very good icing on the cake. In addition to hard materials such as marble, ceramic tiles, and fair-faced concrete, indoor furniture should also be considered to use fabric, wood and textiles that make people feel warm and soft to decorate the space. At the same time, the 
lighting atmosphere can be created to match the overall tone of the space, which can highlight the

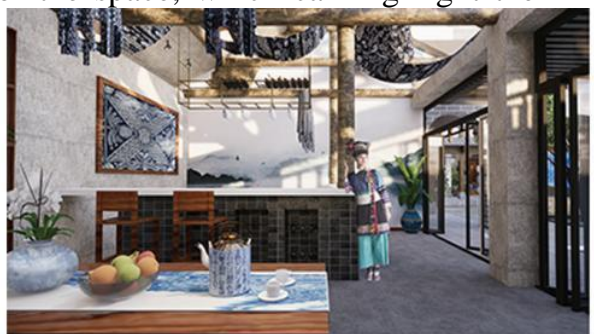

dazzling parts of the interior design.

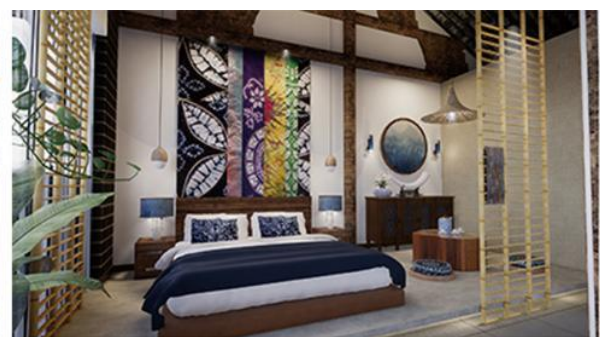

Figure 4 Design of Dali tie-dye theme homestay design renderings of pantry and bedroom design (picture source: the author's own drawing).

\subsection{Principles of Multi-dimensional Application of Patterns}

There are many types of Bai tie-dye decorative patterns, and the patterns are usually simply presented on white cloth in a flat form. They are often used in decorative paintings, sofa backs, curtains, tablecloths and other indoor furnishings in the design of homestays. However, the flat patterns appear relatively thin in space, lacking visual changes and spatial hierarchy. It can be considered to extract several representative tie-dye patterns, refine and streamline them according to the interior design style, and use them as element symbols in the design of homestays to be used and presented in multiple dimensions in the space. For example, the simple geometric patterns that are common in Dali tie-dyeing are simplified, and the patterns can be rearranged, transformed, and reorganized. It can not only be used as decorative elements on walls, ceilings, doors and windows, and furniture surfaces, but also can be three-dimensional. It is used in the design of furniture such as bookshelves, grilles, partitions, and indoor and outdoor furnishings. The multi-dimensional application of Dali tie-dye patterns can be used as a cultural imprint of tie-dye into the hotel space environment to achieve a unified style and rich visual effects.

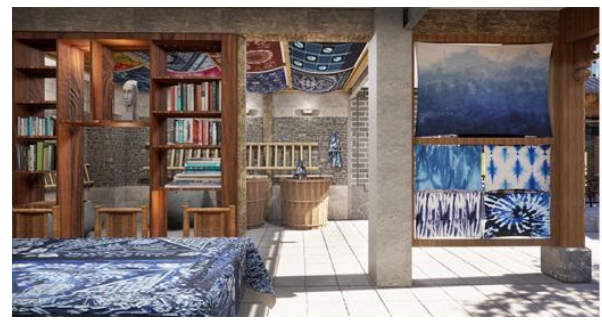

\subsection{The Principle of Experiential and Participatory Space}

While providing accommodation and catering services for tourists, homestays are also a platform for tourists to provide cultural exchanges and cultural experiences. They should fully demonstrate national cultural characteristics and be the inheritors and spreaders of local culture, and the inheritance of culture should not just float Communication at the visual level should enhance the interaction and participation of visitors with culture, and it needs to enrich the experience and interactivity of spatial functions. If an interactive and experiential tie-dyeing workshop can be added to the interior space of the homestay, visitors can have a more intuitive understanding of the tiedyeing skills of Dali and increase the interactivity and interest of the homestay space. ("Figure 5") Integrating regional culture into the homestay design can better protect the local culture and customs of the area and enhance the cultural literacy of the area, so that foreign tourists can better understand the local traditional culture, enhance the awareness of protection, and better inherit the excellent traditional culture and intangible cultural heritage.

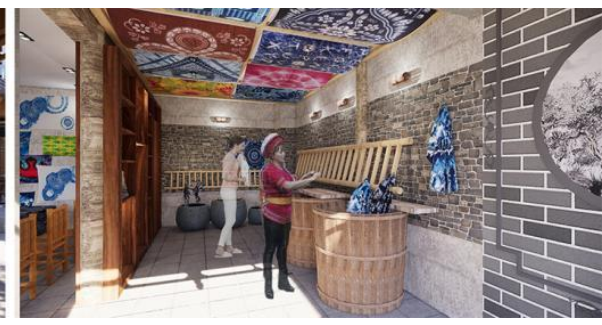

Figure 5 Design of Dali tie-dye theme homestay design effect picture of tie-dye experience workshop (Image source: self-painted by the author). 


\section{CONCLUSION}

The cultural value and national color displayed by the tie-dye of the Bai nationality in Dali are selfevident, and it fits well with the cultural value and regional symbol elements that the design of homestays should emphasize. The combination of tie-dyeing art of Dali Bai nationality and homestay design should consider the unity of space style, the creativity in the use of tie-dyeing elements, and the participation and experience of space. While giving profound connotations to the design of the homestay, it can also contribute to the cultural inheritance and cultural innovation of Dali's tiedyeing skills.

\section{AUTHORS' CONTRIBUTIONS}

This paper is independently completed by Xinyue Wang.

\section{REFERENCES}

[1] Yao Rui, Zhang Haijing. Thoughts on the development of tie-dye technology and characteristic products of the Bai nationality in Dali [J]. Journal of Guangxi University for Nationalities (Natural Science Edition), 2009(S2): 35-37. DOI: 10.16177/j.cnki.gxmzzk.2009.s2.033

[2] Liu Lijuan, Li Xiaorui. The symbolic meaning changes and cultural representation of the tiedyeing craftsmanship of the Bai nationality in Dali [J]. Textile Report, 2021,40(04):97-100.

[3] Han Han. Research on the decoration of tiedye patterns of the Bai nationality in Dali, Yunnan and its application in digital art [D]. Yunnan Art University, 2017.

[4] Tian Shun. A Study on the Architectural Image in the Tie-dyeing Process of the Bai Nationality [J]. Decoration, 2019(07):112-115. DOI : $\quad 10.16272 /$ j.cnki.cn111392/j.2019.07.026

[5] Zhu Xia, Li Xiaocen. Traditional tie-dyeing skills and related issues in Zhoucheng, Dali [J]. Journal of Dali University, 2009,8(09):38-43.

[6] Duan Lifen, Li Chenghong. Inheritance and Development of Artistic Dyeing Technology of Yunnan Minority Nationalities [J]. Dyeing and Finishing Technology, 2020,42(01):52-55. 